\title{
SU ALCUNE MANIFESTAZIONI FUMAROLICHE E SOL- FATARICHE NEL EL SALVADOR (CENTRO AMERICA) $\left(^{*}\right.$ )
}

\author{
Adolfo Perozzi
}

Nel gennaio di questo anno, accompagnando il prof. Francesco Penta in una visita preliminare $\left(^{1}\right)$ della regione salvadorena nel Centro America, ho avuto agio di eseguire alcuni rilievi su diversi campi fumarolici e aree solfatariche in quel territorio. Mi sembra utile comunicare i risultati delle osservazioni effettuate insieme con le poche considerazioni necessarie in proposito.

Studi Preliminari. - Si è presa preventiva visione della letteratura relativa al territorio del Centro America e più in particolare della Repubblica di El Salvador.

Si sono consultati perciò i lavori di G. Mercalli, Sapper, Coleman, Schaffer, A. I. Eardley e le più recenti note già pubblicate da H. Meyer-Abich.

Si è a più riprese esaminata anche la carta geologica in fase di rilevamento gentilmente messa a disposizione dallo stesso dott. MeyerAbich il quale fra l'altro, volta per volta, ha comunicato le sue osservazioni inedite.

In maniera specifica si è presa visione del paragrafo III B (f) dello studio effettuato dalla commissione tecnica dell'UNO intitolato "Development of Geological Resources in El Salvador ".

Sopraluoghi E RiLievi. - Nella mattinata del 14 gennaio 1953, mediante un piccolo aereo messo a disposizione dal Ministro dell'Economia, con il dott. Meyer ed il sig. Tiraboschi sono state compiute due escursioni sopra il territorio della Repubblica per avere una visione di assieme delle zone in cui si sarebbe effettuato il lavoro nei giorni seguenti.

(*) Comunicazione presentata al Convegno dell'Associazione Geofisica Italiana, tenuto a Roma il 17-18 giugno 1953.

(1) Scopo della visita era quello della valutazione dei fenomeni vulcanici esistenti in rapporto alla convenienza o meno di intraprendere ricerche minerarie miranti, a loro volta, alla utilizzazione industriale del vapore acqueo, delle acque termali e termominerali e dei prodotti solidi d'origine solfatarica. 
Nel corso di una prima escursione, partendo dall'aeroporto di llopango (S. Salvador), si è aggirato il vulcano di S. Salvador ed il vulcano di Izalco, attualmente in attività ritmica (vedi foto $1 \mathrm{e} \mathrm{2}$ ), indi rasentando i vulcani di S. Ana, della Laguna Verde, della Lägunila o Ahuachapan si è fatto un ampio giro sui campi fumarolici della zona. Di qui si è risaliti verso il $\mathbf{N}$ compiendo un percorso parallclo

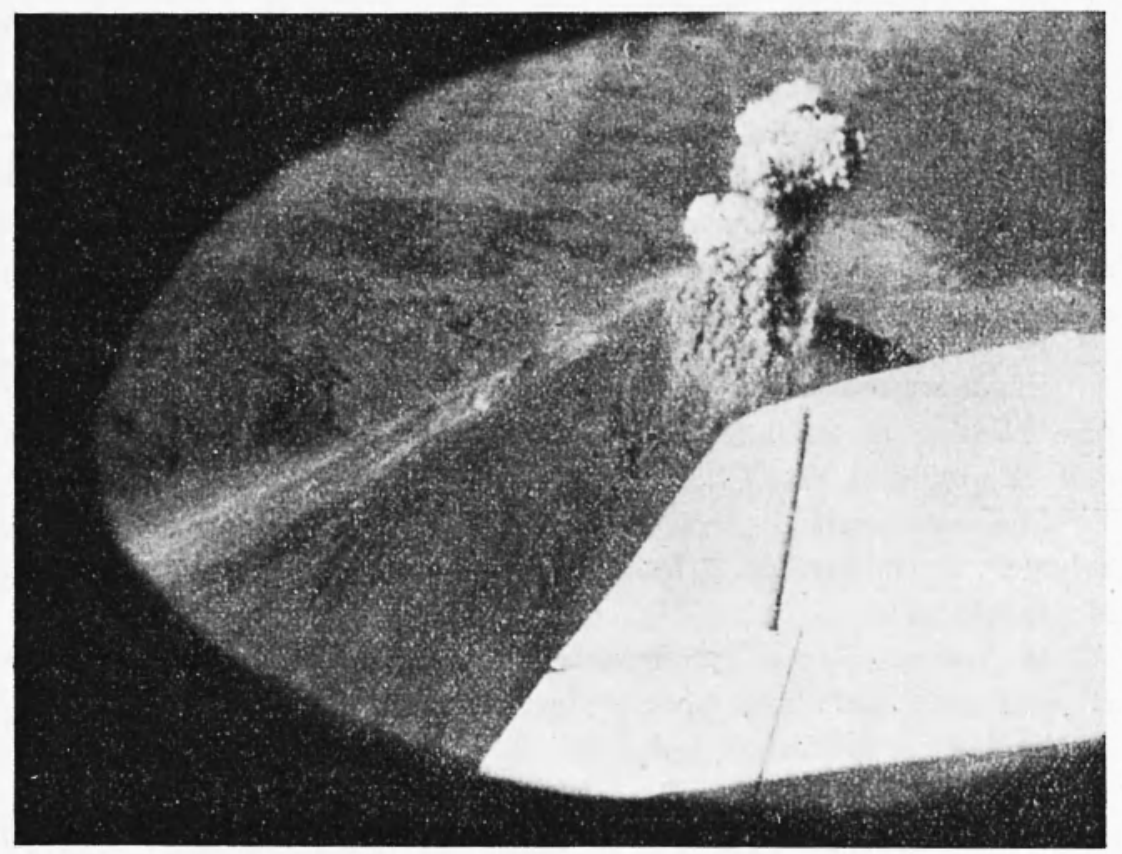

Fig. 1 - Izalco.

alla frontiera con il Guatemala fino a Metapan, alla zona cioc̀ clove affiorano le rocce sedimentarie; da questa localita si è puntato su la Chorrera del Guyabo dove si è virato per fare di nuovo ritorno all'aeroporto.

Durante la seconda escursione è stata sorvolata la parte orientale della Repubblica. Si sono sorvolati i campi fumarolici di S. Vicente, del Tronador, Laguna Alegria di Cbimeca; si è arrivati fino alla città di Union, si è quindi risaliti passando per Carolina, per poi far ritorno all'aeroporto.

Nel pomeriggio del 14 gennaio 1953 è stato effettuato un sopraluogo al vulcano di S. Salvador. Si è potuto constatare lo stato del vulcano dopo l'eruzione del 1917; a distanza, anche nel conetto al 
centro del cratere, non si è constatata attività; non è da escludere però che possano esserci emanazioni di gas sul fondo.

ll 15 gennaio 1953 sono state compiute escursioni ai campi fumarolici di Ahuachapan.

Nella visita sono state guide preziose ed ospitali il Sindaco ed

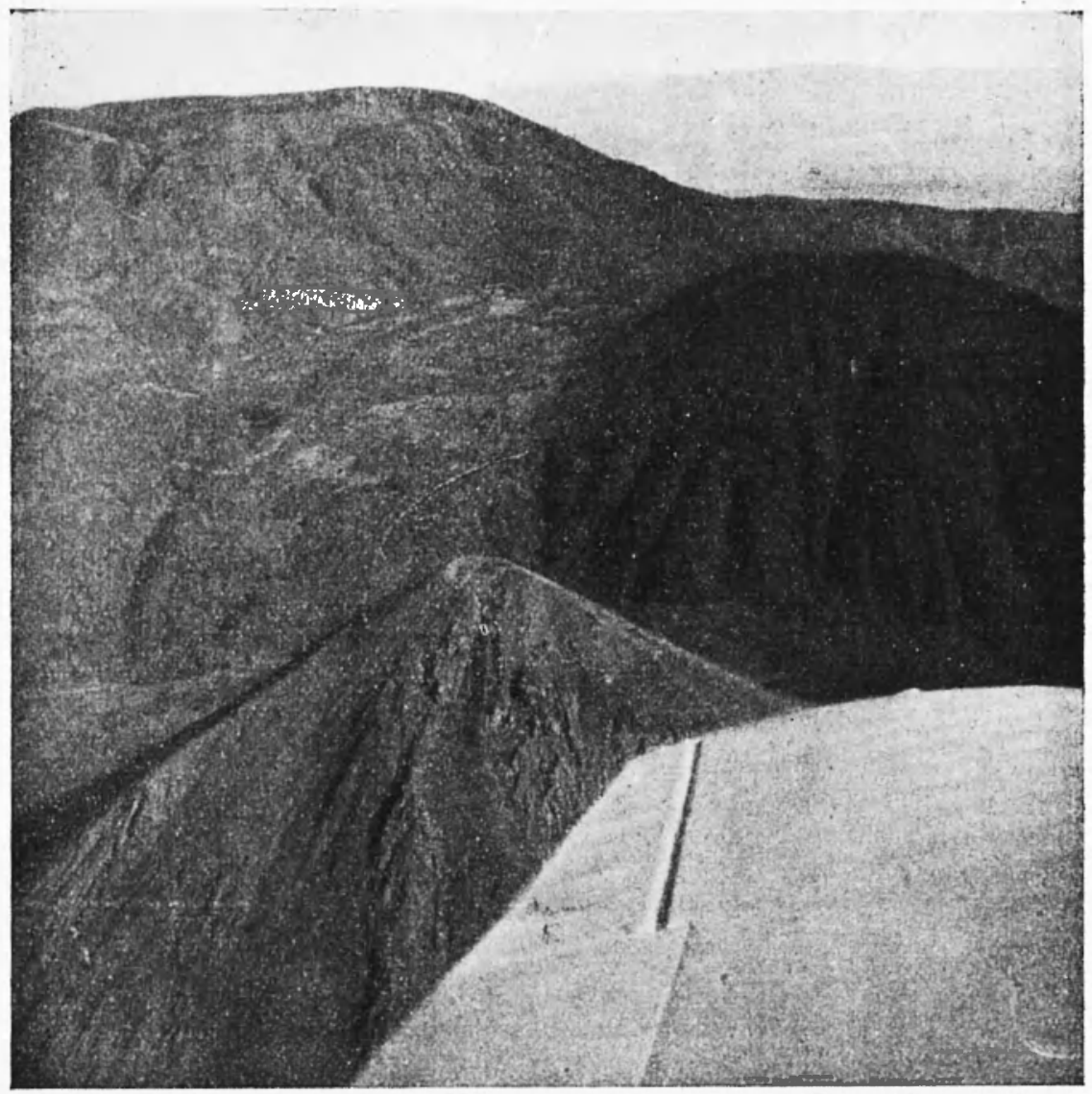

Fig. 2 - Izalco.

il Tesorero Municipale di Ahuachapan, signori José Luciano Betancourt e José Miguel Lainez.

Il 16 gennaio 1953 sono state compiute escursioni ai campi del distretto di Usulutan e di S. Miguel (Chinameca).

Nel pomeriggio del 18 gennaio 1953 si è fatta una escursione a Izalco per effettuare alcune osservazioni sul vulcano. Purtroppo il tempo non è stato clemente ed una spessa nube ha impedito la vista 


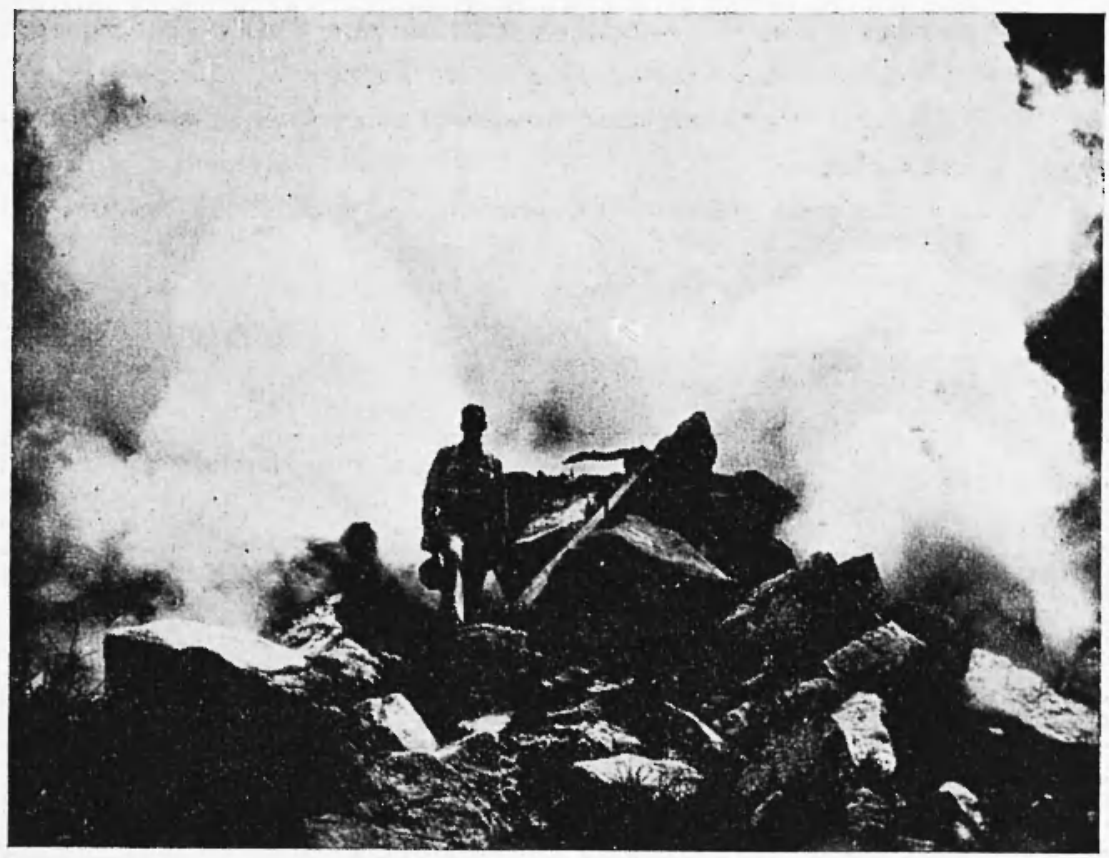

Fig. 3 - Dipartimento di Ahuachapan Ausoles di Cuyanausul.

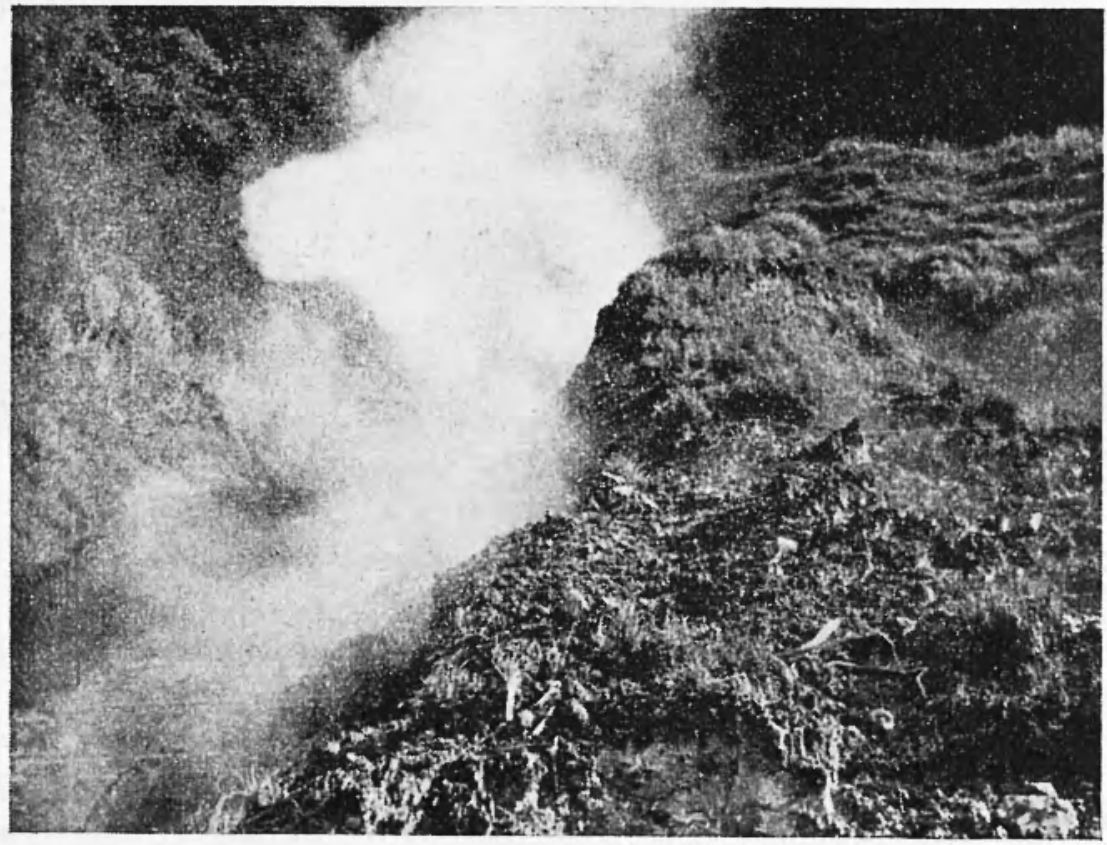

Fig. 4 - Dipartimento di Ahuachapan Ausoles di Cuyanausul. 
delle eruzioni. Si sono potute fare solo poche osservazioni e da lontano.

Il giorno 19 gennaio 1953 è stato compiuto un sopraluogo alla località denominata Los Infernillos di San Vicente con la gentile guida del proprietario del beneficio, dott. Beniamino Iglesias.

Il giorno 20 gennaio 1953 sono state visitate le fumarole di Cuyanausul poste fra le quote 1200 e 1425 ove sono state misurate temperature fino a $125,5{ }^{\circ} \mathrm{C}$.

Esame dei campi fumarolici più Significativi (v. carta d'assieme e cartine dei singoli campi fumarolici). - Si è proceduto alla visita dei campi fumarolici che dall'esame dei documenti a disposizione apparivano più significativi agli effetti dello studio intrapreso e cioè:

A) il gruppo del Dipartimento di Ahuachapan,

$B)$ il gruppo del Dipartimento di Usulutan,

C) il gruppo del Dipartimento di S. Miguel (Chinameca),

D) il gruppo del Dipartimento di San Vicente.

Espongo qui di seguito $e$ in sintesi $i$ risultati delle osservazioni eseguite insieme con il dott. Meyer-Abich e con l'aiuto del chimico Luigi Tiraboschi.

A) Dipartimento di Ahuachapan.

È da notare che nella piana ai piedi dei rilievi vulcanici si rinviene una falda di acqua fredda ad una profondità variabile fra i 30 e grli $80 \mathrm{~m}$ sotto il piano di campagna (notizia fornita dal Sindaco di Ahuachapan).

1) Ausoles de Ahuachapan. $-3 \mathrm{~km}$ circa a SE di Ahuachapan ai piedi del vulcano di Lagunita o Ahuachapan a quota 740

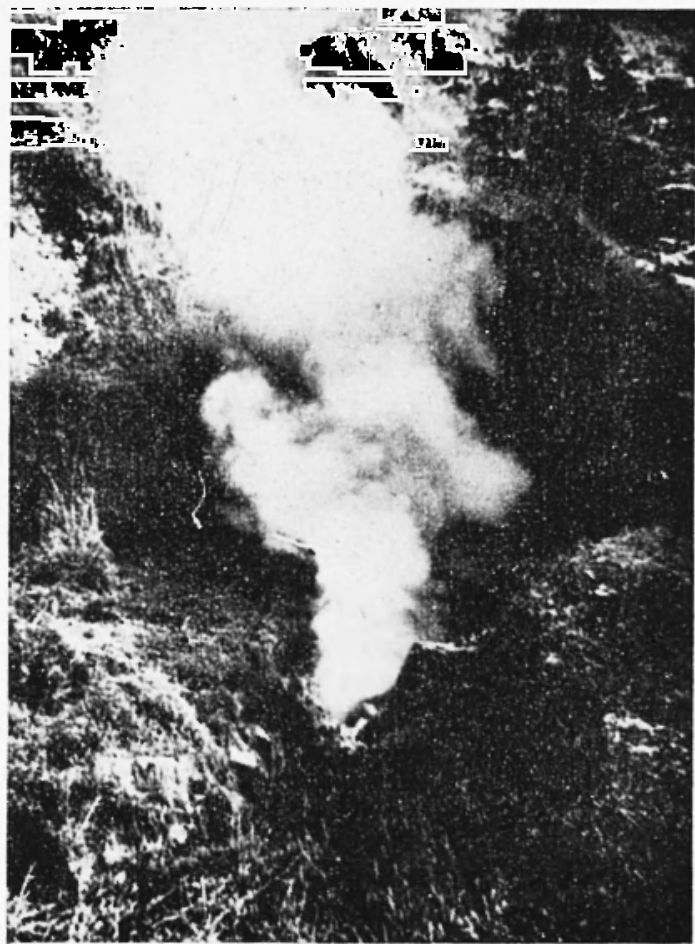

Fig. 5 - Dipartimento di Alıuachapan. Ausoles di Cuyanausul. 
circa, la formazione vulcanica è incisa dal Rio Agua Calicnte. Il terreno appare "fumarolizzato » nel letto del fosso, sulle pareti dell'incisione operata dal fosso stesso e sull'attiguo pianoro ad $\mathbb{W}$, il vapore acqueo esala da numerose buche; si ode ovunque il borbottio dell'ebollizione

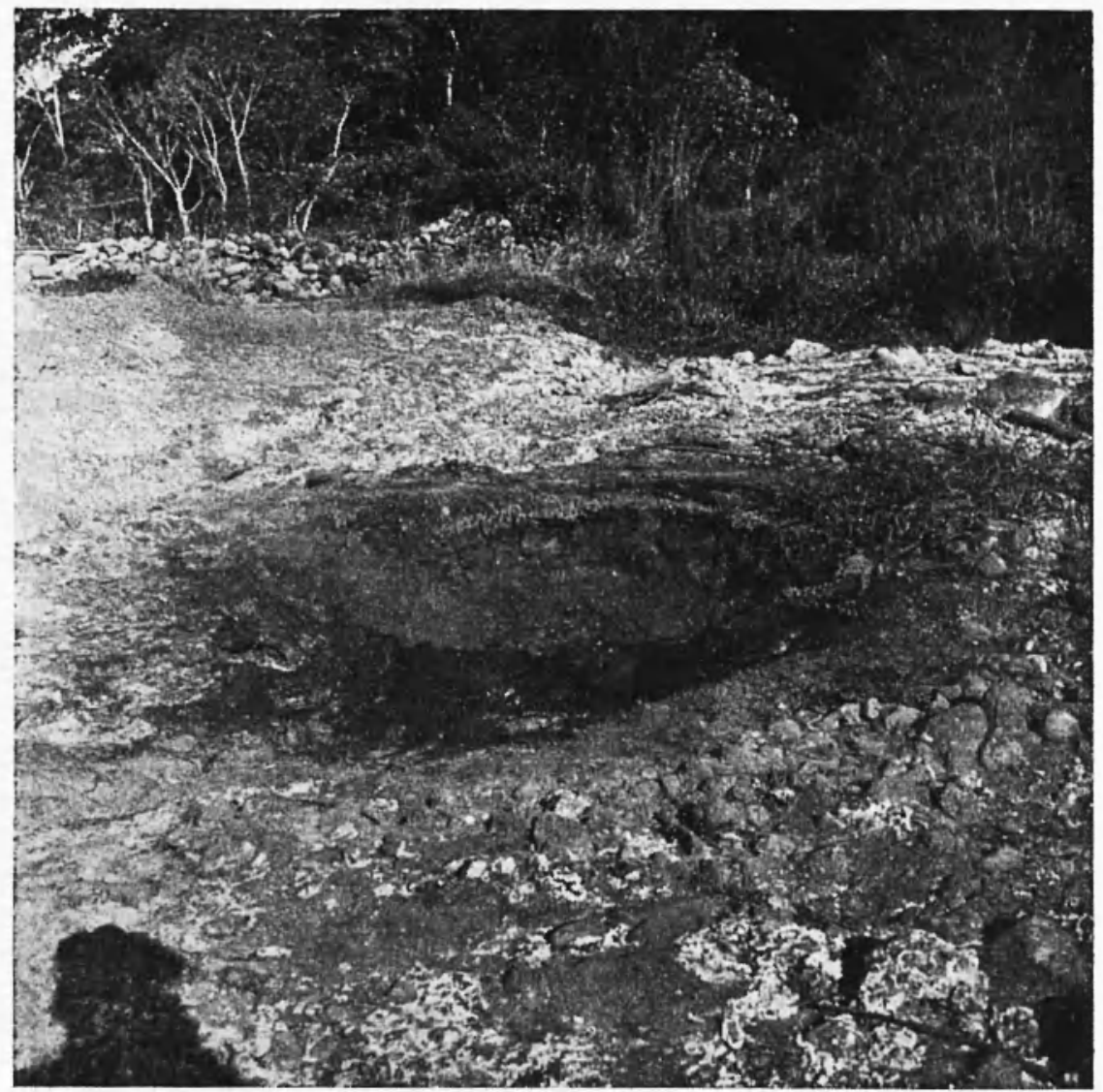

Fig. 6 - Ausoles de Agua Cluca. Buca-conetto di fango bollente.

delle acque sotterranee a poca profondità. Intorno alle emergenze attive o no si notano incrostazioni di cloruri, di idrossidi di ferro, di zolfo ecc. Nelle esalazioni di $H, O$ è anche constatabile la presenza di $\mathrm{H}_{2} \mathrm{~S}$ e $\mathrm{CO}$.

Le temperature massime misurate entro le buche esalanti vapore sono state di $99^{\circ} \mathrm{C}$.

2) Ausoles de "La Labor ". - Prossime all'omonimo " beneficio " di caffè e zucchero. Le manifestazioni presentano gli stessi caratteri di 

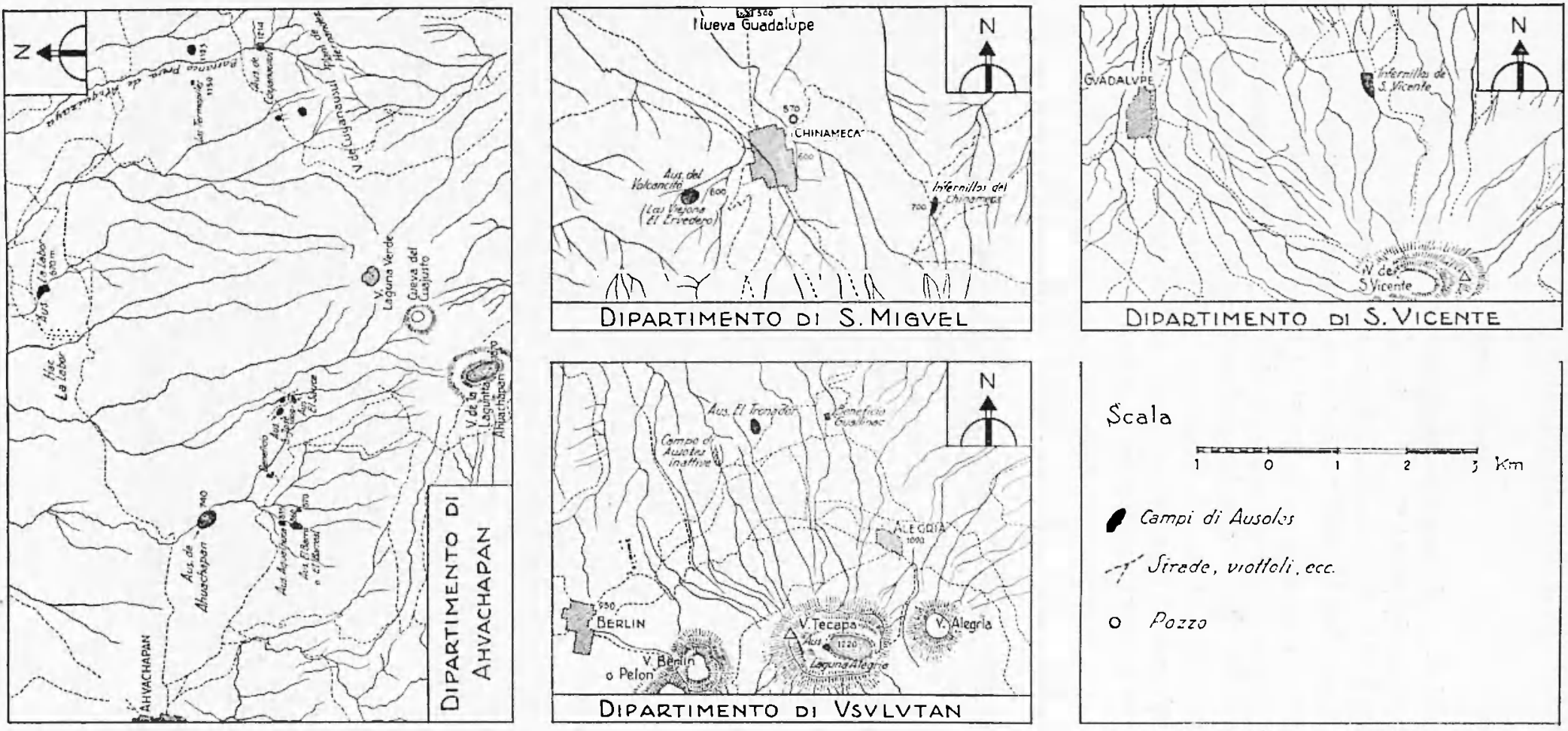

Cartina dimostrativa dei principali campi fumarolici. 
quelle del n. 1 ed hanno la loro sede nel fondo e sui fianchi di un avvallamento imbutiforme del terreno aperto verso $N$. Sulla soglia di questa porta affiora una colata lavica con struttura a piastrelle. Sul fondo e sui fianchi dell'avvallamento affiorano prodotti piroclastici con grossi blocchi di lava. La quota delle manifestazioni è circa $670 \mathrm{~m}$. Le temperature massime misurate di $99,5{ }^{\circ} \mathrm{C}$. Anche in questa area si notano gli effetti della fumarolizzazione del terreno e si sente il borbottio delle acque in ebollizione nell'immediato sottosuolo. Secondo informazioni assunte sul posto, nelle vicinanze di questo campo di ausoles esistono sorgentelle di acqua non termale.

3) Ausoles de "Agua. Chuca " (Canton El Barro e El Barreal). Comprendono tre campi di ausoles. Uno a quota 860 , un secondo a

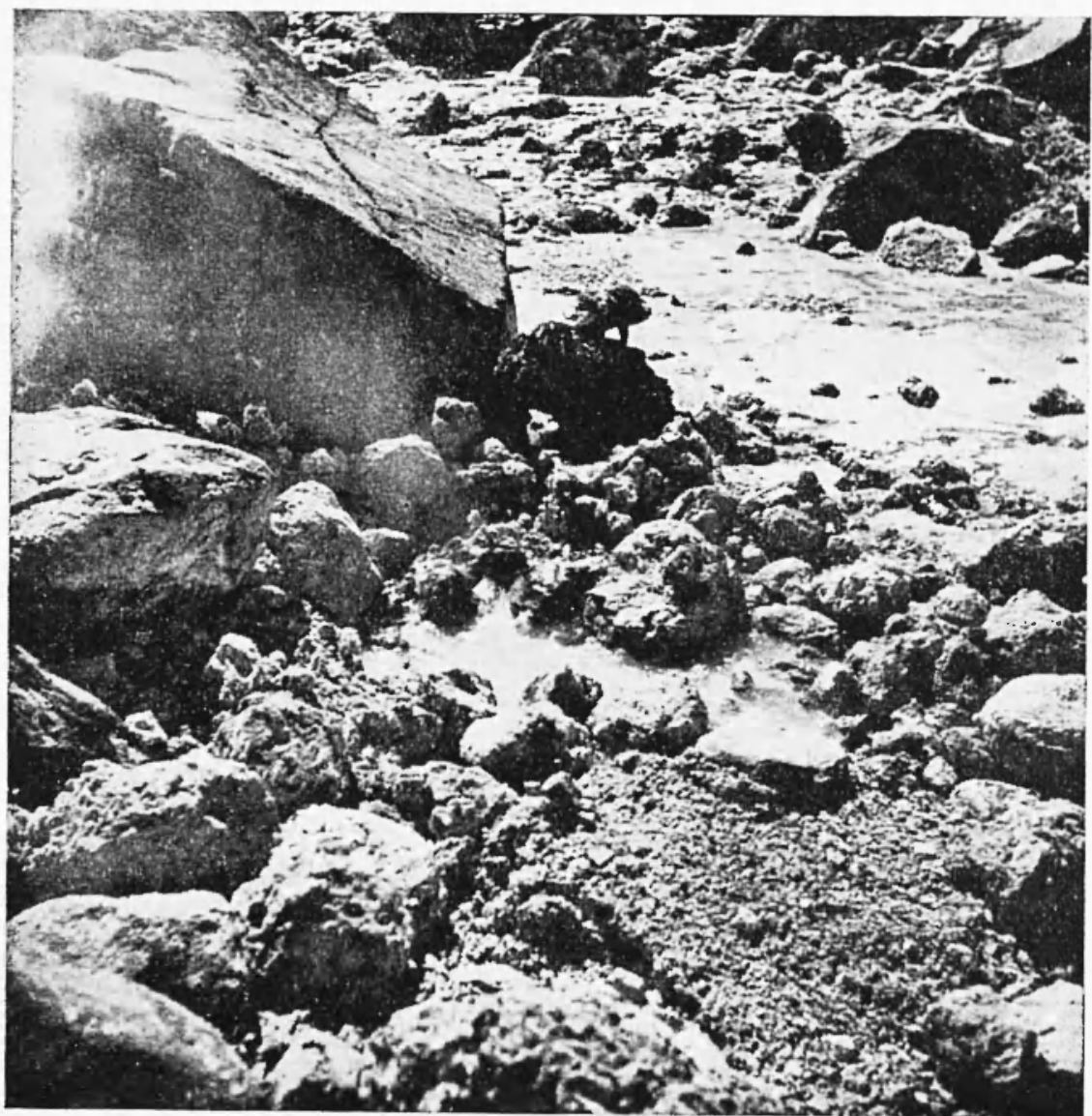

Fig. 7 - Ausoles di Ahuachapan. L'aequa ribolle fra $i$ massi. 
quota 835 allineati lungo una direttrice N-S e distanti fra di loro circa $300 \mathrm{~m}$. Nelle aree in esame esistono buche di vari metri di diametro dalle quali esala vapore acqueo. Per lo più sul fondo delle buche ribolle del fango con formazione spesso di piccoli conetti di fango. Le temperature massime misurate sono state di $96^{\circ} \mathrm{C}$. In vicinanza del campo a quota 835 scorre un ruscello la cui acqua ha una temperatura di $35{ }^{\circ} \mathrm{C}$ (temp. dell'aria ambiente $2 \tau^{\circ} \mathrm{C}$ ).

Un terzo piccolo campo di ausoles si incontra a quota 870 circa, qualche centinaio di metri più ad Est del precedente; esso è costituito principalmente da una grossa buca fra blocchi di lava dal fondo della quale fuoriesce vapore a temp. di $98{ }^{\circ} \mathrm{C}$.

4) Ausoles "El Sauce" e "San José ». - Si estendono su di un'area di $400 \times 200 \mathrm{~m}$ circa da ambo i lati della strada a quota 1000 circa. Affiorano anche qui nell'intorno delle manifestazioni grossi blocchi di lava. L'acqua ribolle nel snttosuolo dando luogo a correnti di vapore ed a piccole vasche $\mathrm{di}$ fango. La temperatura massima riscontrata ̀̀ stata di $97^{\circ} \mathrm{C}$. Più ad Est ed a quota più al. ta si incontrano le sorgenti d'acqua potalbilc che alimentano l'acquedotto di Ahuachapan (secondo le informazioni del Sindaco.

5) Ausoles de Cuyanausul. - Sotto questa denominazione si rarcoløono 4 campi di ausolcs localizzati lungo due barrancos adiacenti.

I primi due hanno le loro sedi lungo il harranco denominato

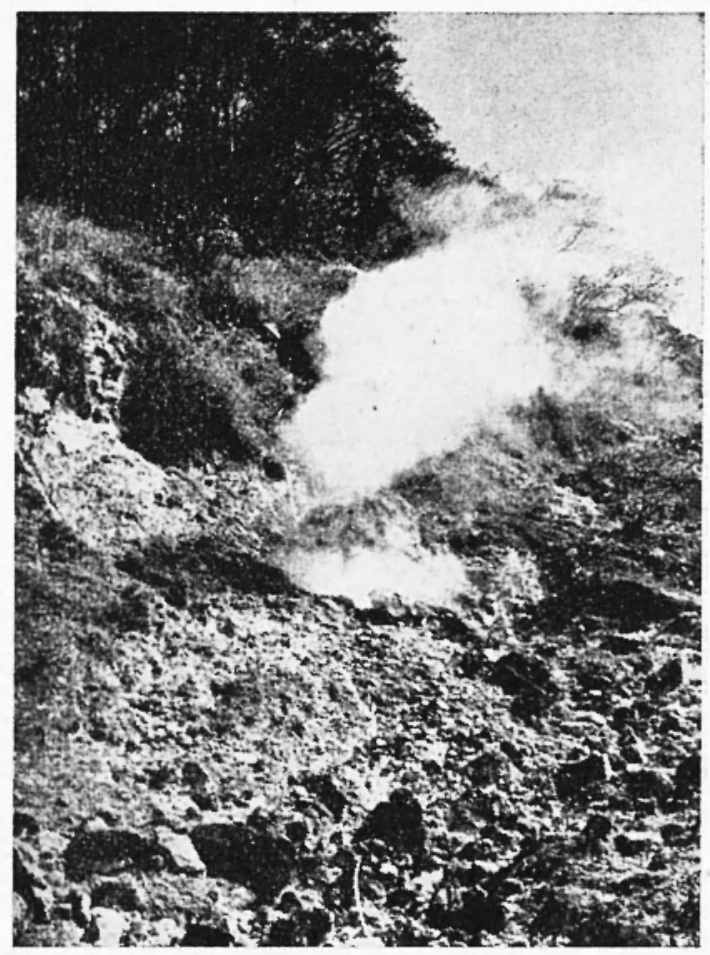

Fig. 8 - "El Tronador ». Si noti come il pennacchio di vapore non è visibile all'uscita trattandosi di vapore secco $\left(107^{\circ} \mathrm{C}\right)$. 
"Presa de Atiquizaya ". Il campo più alto a 1.200 m s.l.m. si sviluppa lungo il Rio le cui acque a monte delle manifestazioni hanno una temperatura di $17^{\circ} \mathrm{C}$ (temp. aria $20^{\circ} \mathrm{C}$ ). Il vapore, ac! una temperatura massima di $97{ }^{\circ} \mathrm{C}$ esce da numerosi punti ed è accompagnato da $H . S$ ed altri gas.

Il campo più basso, a circa $1115 \mathrm{~m}$ s.l.m. sul fianco Est del harranco, è più esteso. Come anche nel precedente si ode l'acqua ribollire nel sottosuolo. La massima temperatura misurata $\grave{e}$ stata anche qqui di $97^{\circ} \mathrm{C}$. In questo campo si è notata una notevole quantità di zolfo aciculare in bei cristalli lunghi fino a $2 \mathrm{~cm}$ specie intorno alle piccole buche del terreno da cui scaturisce il vapore.

I secondi due campi fumarolici hanno le loro sedi nel barranco

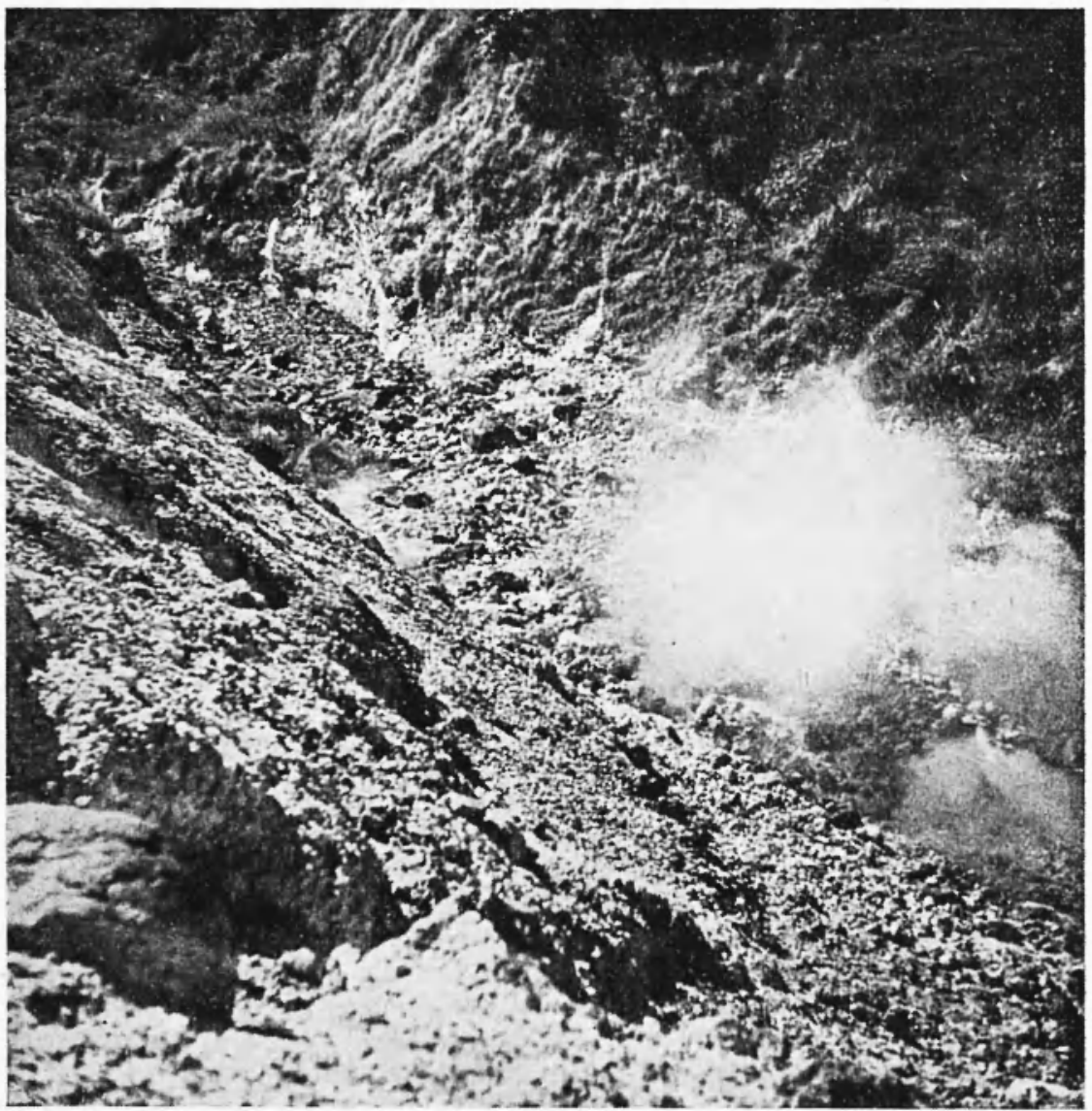

Fig. 9 - Las Infernillos de S. Vicente. 
attiguo ad $\mathbb{W}$, harranco che sembra fra le quote dei due campi di ausoles inciso in una unica od in una serie di colate laviche. In ambedue questi campi la quantità di $H_{2} S$ è piccola, come si può constatare dall'odore e dalla mancanza quasi di incrostazioni di zolfo nelle adiacenze delle ausoles. In ambedue le portate di vapore emesso sono notevoli e la temperatura alta; sono stati infatti misurati $112{ }^{\circ} \mathrm{C}$ nel campo di ausoles a $1436 \mathrm{~m}$ e fino a $125,5{ }^{\circ} \mathrm{C}$ nel campo a $1280 \mathrm{~m}$ s.l.m; date le notevoli difficoltà incontrate nelle misure, non si può affermare che queste temperature siano effettivamente le massime. A $1370 \mathrm{~m}$ si trova una piccola captazione di acqua termale a $47{ }^{\circ} \mathrm{C}$.

\section{B) Dipartimento di Usulutan.}

1) Ausol "El Tronador". - Il gruppo detio del Tronador ha la sua sede a quola 800 circa in un barranco ad W della strada che la Mercedes Umaña porla a Berlin. Nell'incisione affiorano grossi blocchi di rocce vulcaniche (fino a qualche mci. Piu in alto lungo la strada affiorano prodotti piroclastici fumaro!izzati e un banco di lava. Al centro della valletta dove essa si spiana fra i blocchi si ode il borbottio dell'acqua che bolle; la massima temperatura misurata è stata di 96 "C.

Sul piccolo pianoro esistono ancora le rovine di una piscina ed i resti di tulazioni.

Sul fianco $N$ della incisione, una trentina di metri più alta del pianoro, vi è una fuoriuscita sibilante di vapore secco a 107 "C. In tutta l'area la superficie è ricoperta da incrostazioni di sali, per lo più cloruri, di zolfo, da zeoliti ecc. È rla notare che le tracce di alterazioni si prolungano in alto fino alla incisione creata dalla sirada rotalile: attualmente, però, l'attività fumarolico-solfatarica è limitata alla parte hassa e cioè sulla parete dell'incisione e sulla spianata ove sono i resi della vasca. Nel complesso le manifestazioni sono parzialmente collegate ad acqua dell'immediato soltosuolo, forse anche di scaturiggine di una vena soiterranea proveniente dalle montagne sovrastanti; però la temperatura $\left(107{ }^{\circ} \mathrm{C}\right)$ delle esalazioni sscche rende molto verosimile l'ipotesi che gli spiragli vulcanici primari sepolti non siano lontani.

2) Ausoles de la Laguna de Alegria a q. 1220 sul l.m. nell'interno del cratere del vulcano Tecapa. Sul bordo occidentale del lązo che occupa il centro del cratere il terreno si presenta fumarolizza:o. Da 
alcune buche nel terreno geme del vapore a $96^{\circ} \mathrm{C}$. Il paesaggio ricorda quasi in tutto la Solfatara di Pozzuoli (Napoli).

\section{C) Dipartimento di S. Miguel (Chinameca).}

1) Ausoles "El Infernullo 》 de Chinameca. - Poste in una incisione che attraversa la strada circa $4 \mathrm{~km}$ ad Oriente di Chinameca. Le esalazioni di vapore si verificano attraverso piccole buche sul fian-

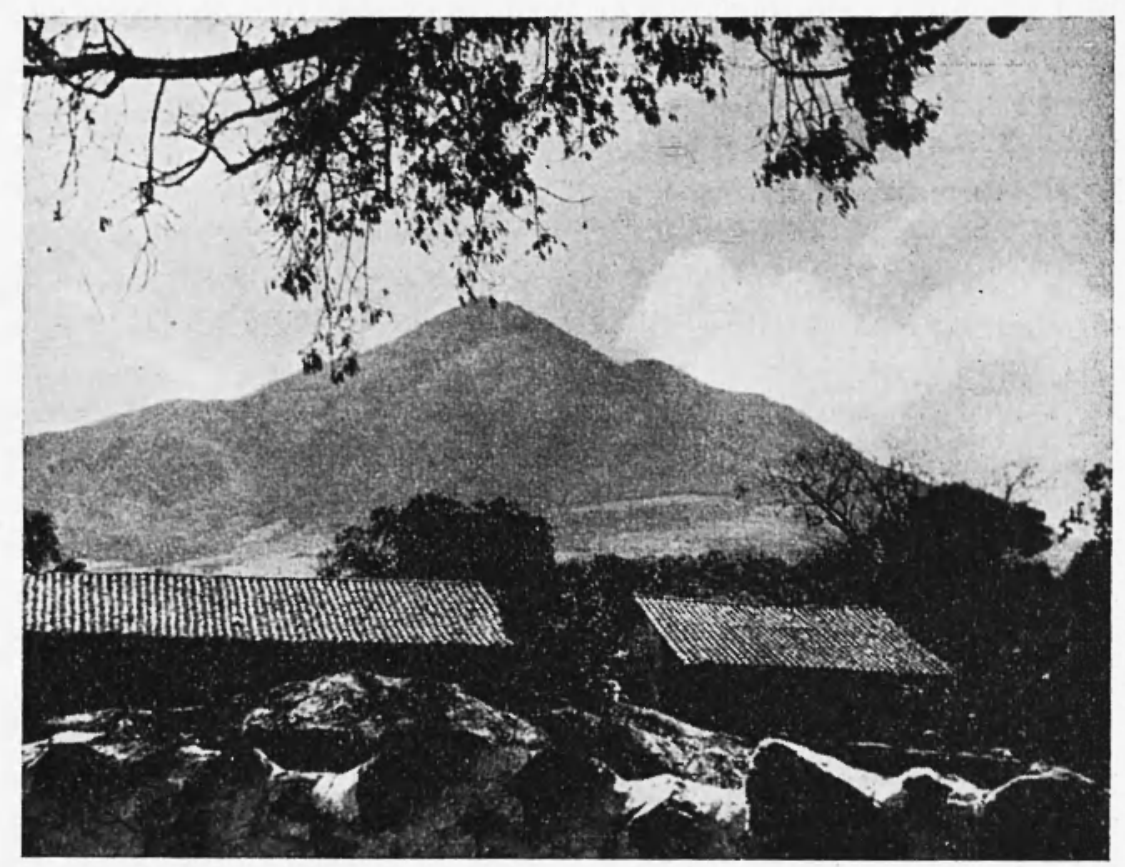

Fig. 10 - Il vulcano di S. Vicente.

co orientale della incisione, alveo di un piccolo corso d'acqua, il quale era completamente asciutto al momento della visita. Sulla roccia si depositano zolfo, cloruri ecc.; secondo le osservazioni del dott. Meyer-Abich, lungo la direttrice della suddetta incisione (NE) si hanno tre bocche di piccoli vulcani relativamente recenti. La quota della località è circa $700 \mathrm{~m}$, la temperatura massima registrata nella buca esalante vapore è stata di $99,5{ }^{\circ} \mathrm{C}$. All'atto della visita le manifestazioni idriche calde e bollenti sembravano potersi interpretare anche come sorgenti : affioramenti cioè delle falde freatiche o forse in parte anche della corrente subalvea. 
2) Ausoles "El Vulcuncito » (la Viejona). - Le manifestazioni si incontrano sulla falda Nord del "Vulcancito " lungo un fosso diretto per WNW-ESE. Attraverso i piccoli fori da cui fuoriesce vapore ed acqua si ode il caratteristico borbottio dell'acqua in ebollizione. La località è a quota 600 circa; la temperatura massima misurata nei punti di ebollizione e di esalazione è stata di $99{ }^{\circ} \mathrm{C}$. Il fosso al momento della visita era asciutto. Non si può escludere che l'acqua bollente con il relativo vapore che affiora qua e là sotto il fondo sia la corrente subalvea del fosso stesso. Tale corrente può però coincidere anche con la falda freatica.

$\grave{E}$ opportuno ricordare che vicino all'abitato di Chinameca in prossimità di una colata basaltica potente $75 \mathrm{~m}$ (Flan de Las Mesas) è stato scavato a mano un pozzo profondo $100 \mathrm{~m}$ circa (il piano di campagna è $550 \mathrm{~m}$ circa sul $1 . \mathrm{m}$.); sul fondo è stata incontrata una falda d'acqua a temperatura di $38{ }^{\circ} \mathrm{C}$ circa. Detta acqua al gusto ed all'olfatto non presenta particolarità. Per questo pozzo vedasi la memoria a stampa di Meyer-Abich.

\section{D) Dipartimento di San Vicente.}

Los Infernillos de San Vicente. - Il campo di ausoles visitato ha la sua sede sul fondo e sui fanchi di un barranco circa $3 \mathrm{~km}$ ad Est di Guadalupe ed a circa $820 \mathrm{~m}$ sul l.m. Sui fianchi del harranco affiora un hanco di lava alterata e fumarolizzata. Le manifestazioni principali sono raccolte sul fondo del rio. La temperatura massima misurata è stata di $99{ }^{\circ} \mathrm{C}$. Circa $150 \mathrm{~m}$ a monte di questa zona esiste una piccola sorgente fredda.

Considerazioni. - Nei dipartimenti visitati e che sembrano essere quelli più ricchi di manifestazioni esalativo-vulcaniche importanti, le temperature massime note dalla letteratura erano rispettivamente: per $A 981 / 2{ }^{\circ} \mathrm{C}$; per $B$ (Tecapa) $27-28{ }^{\circ} \mathrm{C}$; per $C 981 / 2{ }^{\circ} \mathrm{C}$; per $D 98{ }^{\circ} \mathrm{C}$.

Con le misure da noi effettuate si sono riscontrate temperature per lo più superiori, alcune decisamente superiori a quelle precedentemente note; al Tronador infatti la temperatura è risultata (il 16 gennaio 1953 ) $107{ }^{\circ} \mathrm{C}$ ed a Cuyanausul $125,5{ }^{\circ} \mathrm{C}$ (il 20 gennaio 1953). Le nostre misure di temperatura sono state effettuate circa 2 mesi dopo la fine della stagione delle pioggie.

Se a Sapper non sfuggirono i punti nei quali si sono riscontrati 
i massimi rispettivamente di $107^{\circ} \mathrm{C}$ e di $125,5{ }^{\circ} \mathrm{C}$, le sensibili differenze trovate potrebbero avere più di una spiegazione; $\grave{e}$ anche possibile un aumento della termalità in generale. Una parola decisiva, però, non può dirsi senza aver ripetuto le misure molte volte ed in periodi climatici diversi; in ogni caso conviene istituire delle misure sistematiche e periodiche.

Ricerche queste che impianterà e condurrà il dott. Meyer-Abich della Università di S. Salvador.

Nel chiudere questa nota esprimo i ringraziamenti al Governo Salvadoreño, ed in particolare al dott. Jorge Sol Castellanos, Ministro dell'Economia, per la cordiale ospitalità, per l'occasione fornitami di visitare un cosi interessante Paese e per la gentile autorizzazione data alla pubblicazione della presente nota.

Roma (S. Pietro in Vincoli) - Istituto di Giacimenti Minerari e di Geologia Applicata dell'Università — Giugno 1953.

\section{RIASSUNTO}

Si descrivono brevemente le manifestazioni fumaroliche e solfatariche più importanti della Repubblica di El Salvador nel Centro America, quelle ciò̀ che hanno le loro sedi lungo una fascia che passa per $i$ vulcani ancora attivi e a ricordo storico di Izalco, S. Salvador, Ilopango, S. Vicente, S. Miguel.

Le maggiori temperature sono state misurate a Cuyanausul (Dipartimento di Ahuachapan) con $125,5{ }^{\circ} \mathrm{C}$ ed al Tronador (Dipartimento di Usulutan) con $107{ }^{\circ} \mathrm{C}$. Esse sono molto maggiori di quelle note dalla letteratura che sono sempre inferiori a $100^{\circ} \mathrm{C}$.

Si avanza l'ipotesi che ciò sia dovuto ad un aumento della termalità in generale della regione, a meno che le bocche con temperature più alte non siano sfuggite ai precedenti ricercatori perché meno appariscenti esalando da esse vapore secco.

\section{SUMMARY}

The A. dealts with the most important fumaroles and solfataric activity which exists in the Republic of El Salvador (Central America). The solfataric fields are spread within a strip which contains both 
active and quiescent volcanoes like Izalco, S. Salvador, Ilopango, S. Vicente etc.

The highest temperatures, which have been recorded at Cuyanausul (Auachapan Dept.) and at El Tronador (Usulutan Dept.), reach 125.5 ${ }^{\circ} \mathrm{C}$ and $107.0^{\circ} \mathrm{C}$ respectively; the actually recorded temperatures are therefore higher than it was formerly reported (less than $100^{\circ} \mathrm{C}$ ).

The $A$. supposes this raise may be related with a general increase of the thermality of the region; it is also possible however, the hottest vents had been overlooked from the previous surveyors, for they produce almost dry steam, and they are therefore less showy than the warmer. 